\title{
The Mechanical Deformation Research of the Annular Ring Based on the Function of the Device of "Abandoning Lines to Protect Electric Poles"
}

\author{
XIAO Kai, ${ }^{1, a}$ HU Qingchun ${ }^{2, b}$, LI Wensheng ${ }^{1, c}$, \\ LIN Zhisheng ${ }^{2, d}$, NIE Ming ${ }^{1, e}$, WEN Jiaqi ${ }^{2, f}$ \\ ${ }^{1}$ Guangdong Power Grid Corporation Electric Power Research Institute, Yuedian Mansion, 8 \\ Shuijungang, Dongfeng East Road, Yuexiu District, Guangzhou, China \\ ${ }^{2}$ School of Mechanical and Automotive Eng, South China University of Tech, 381 Wushan Road, \\ Tianhe District, Guangzhou, China \\ a xk0606@sina.cn, b huqc@scut.edu.cn, ${ }^{\mathrm{c}} 21535639 @ q q . c o m$, \\ d824414996@qq.com, êadminnie@163.com, ${ }^{\dagger}$ wankaki@163.com
}

\begin{abstract}
Keywords: Abandoning lines to protect electric poles; Annular ring; Finite element analysis; Mechanical deformation; Experiment in mechanics

Abstract. The device named "abandoning lines to protect electric poles" was mainly used for the weak links of the system of distribution network lines to motion first when the design wind speed was oversize, and the core components of the device were made up with a cutting edge shaft and an annular ring locked above. By making a study about the deformation of the ring under the load, it got the displacement function and the maximum deformation based on the process of "abandoning lines to protect electric poles". By changing the relevant geometric parameters of the annular ring, it got different curves of displacement function. Optimize the design of the annular ring by finite element analysis and it showed that the smaller of the inner diameter and the larger of the cross section area would get the smaller stress and deformation. After building the experiment in mechanics, the changing details of force was arrived and it verified that the scheme was feasible, finally, by presenting some points for designing the transitional step of the cutting edge shaft, it provided the reference basis of the device of "abandoning lines to protect electric poles".
\end{abstract}

\section{Introduction}

It is very significance for the distribution network lines to create social economic value and meet power supply to the households, so the security of power system has been regarded more and more important now[1]. Now the safety operation of distribution network lines mainly focuses on the layout and reinforce of the poles, but lack in the actively protecting[2].

Rupture and collapse of the poles are serious accidents. In 2013, the Guangdong province suffered from the heavy typhoon Usagi, which made 15635 poles collapse and more than 177 billion yuan lost. Therefore, the China Southern Power Grid Company Limited had come up with an idea, that is "abandoning lines to protect electric poles". It means that make the weak links motion first by itself when there is overload in the distribution network lines system, which are made up with poles, lines, fitting and porcelain crossarm, so as to avoid repairing the expensive parts, especially the poles.

Therefore, so many enterprises and researcher had developed some related devices and realized them. For example, the Guangzhou Kaineng Electric Industrial co., LTD had developed the Connecting Clamp Scheme. It set the weak links in the lines. After installing the connecting clamp, it cut off the line and controls the clamp force between the clamp and the lines, and then when the wind speed exceeds the designed one, the lines would fall off from the device, so the pole would no suffer the load from the line more, in this way, the pole would be protected[3]. Besides, Guangdong Power Grid Corporation Electric Power Research Institute had developed the integration device of Energy absorption to abandon lines to protect electric poles, which can protect lines and poles through a gap in the elastic frame jig when wind is light and absorbed energy to abandon lines but keep pole safety when the wind is heavy[4]. In addition, the China Southern Power Grid Company Limited had developed 
some scheme, such as the Shearing Bolt, Spring Take off Line Device and Integration of Two Phase Load Device. All of the devices above had been installed in the distribution network line and being test.

However, the Connecting Clamp Scheme needed to cut off the lines so it would result the bad current, and it was hard to assemble, which makes the low precision. For the Spring Take off Line Device, because the spring was easy to disturbance under the external environment, it would cause the malfunction or false operation. So the schemes above were not very good.

This paper came up with one new device of "abandoning lines to protect electric poles", which was shown in Figure 1. It consisted of the whole device on the left and the core component on the right. The core components were made up with the cutting edge shaft and the annular ring covered above. At first, the annular ring was covered closely on the small shaft end, when the distribution network lines were overload, the force transmitted from the line davit to the cutting edge shaft, so the annular ring would be extruded by the shaft and deform. Because of the limit position in the shaft by the shell, the annular ring could only step over large end of the cutting edge shaft, in this way, the cutting edge shaft would move some distances, which lead the line davit lose one side support and then the line would fall off from the device, so it realized abandoning lines to protect electric poles.
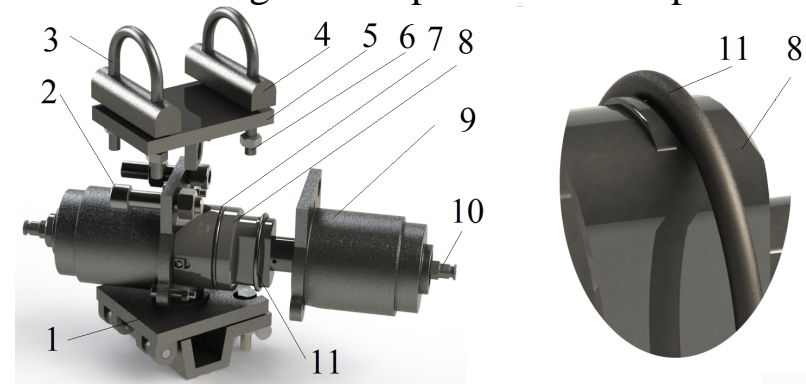

1.Line davit 2.Shell 3.U-hoop 4.Raising block 5.The connecting-converting device 6. Threaded fastener 7. O type sealing ring 8.Cutting edge shaft 9.shell 10. check valve 11.annular ring

Fig.1 Device for Abandoning Lines to Protect Electric Poles from Overload with Multi-Direction in Transmission and Distribution Lines

The size of the annular ring was the most critical factor to affect the function of the "abandoning lines to protect electric poles" scheme. The paper analyzed the deformation process of the annular ring by theoretical analysis, finite element analysis and mechanics experiments, which provided the reference frame to develop the "abandoning lines to protect electric poles" scheme.

\section{The Theoretical Analysis of Annular Ring under Partial Load}

\section{The Model Building of Annular Ring.}

Choose the annular ring to analysis, the model building was shown in Figure 2.

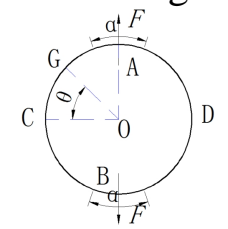

Fig.2 The Diagram of the Model Building of Annular Ring under Load

As the annular ring was centrosymmetric in geometry, its deformation under the load was centrosymmetric, too. As above, Point A,B,C and D were uniformly distributed, among them, A and B were the loading contact areas between the annular ring and the cutting edge shaft. Set the distributed load as $q$ and the central angle of contact arc as $\alpha$, also, C and D were the gap positions in the cutting edge shaft and $\mathrm{G}$ was any point on the ring. Choosed quarter of the ring to analysis below[5]. 
The Calculation of the Deformation of Annular Ring under the Load.

The paper caculated the deformation of the annular ring based on the Castigliano's second theorem, the formula was shown below[6].

$$
\delta_{i}=\frac{\partial U}{\partial F_{i}}=\int_{l} \frac{F_{N}(x)}{E A} \cdot \frac{\partial F_{N}(x)}{\partial F_{i}} d x+\int_{l} \frac{M(x)}{E I} \cdot \frac{\partial M(x)}{\partial F_{i}} d x .
$$

Among them, ${ }^{\delta_{i}}$ was the generalized displacement of Point $i, \mathrm{~m} ;{ }^{F}$ was generalized force, $\mathrm{N}$; $F_{N}$ was axial force, $\mathrm{N} ; M$ was bending moment, $\mathrm{N} \cdot \mathrm{m} ; E$ was elasticity modulus, pa; $A$ was cross sectional area, $\mathrm{m} 2 ; \quad I$ was inertia moment,

$\mathrm{m} 4$, and $I=\frac{\pi \cdot d^{4}}{64} ; d$ was section radius, $\mathrm{m}$.

The force and couple of Point $\mathrm{G}$ on the quarter of the ring was got by Unit-load Method, which is illustrated below[7].

$$
\begin{array}{ll}
F_{N}(\phi)=F_{C} \cos \phi, & M(\phi)=F_{C} R(1-\cos \phi)+M_{C}, \\
F_{N}^{0}(\phi)=\frac{\partial F_{N}(\phi)}{\partial F_{C}}=\cos \phi, & M^{0}(\phi)=\frac{\partial F_{N}(\phi)}{\partial F_{C}}=R(1-\cos \phi), \\
F_{N}^{0}(\phi)=\frac{\partial F_{N}(\phi)}{\partial M_{C}}=0 ; & M^{0}(\phi)=\frac{\partial F_{N}(\phi)}{\partial M_{C}}=1 .
\end{array}
$$

Considering the influence of distributed load $q$, it got the displacement $\delta_{\theta}$ of Point G.

$$
\delta_{\theta}=\left\{\begin{array}{cc}
\int_{0}^{\theta} \frac{0.5 F \cos \phi}{E A} \cdot \cos \phi \cdot R d \phi & \left(0<\theta \leq \frac{\pi}{2}-\frac{\alpha}{2}\right) \\
& +\int_{0}^{\theta} \frac{0.5 F R(1-\cos \phi)+M_{C}}{E I} \cdot R(1-\cos \phi) \cdot R d \phi \\
\int_{0}^{\frac{\pi}{-}-\frac{\alpha}{2}} \frac{0.5 F \cos \phi}{E A} \cdot \cos \phi \cdot R d \phi & \\
+\int_{0}^{\frac{\pi}{2}} \frac{\alpha}{2} \frac{0.5 F R(1-\cos \phi)+M_{C}}{E I} \cdot R(1-\cos \phi) \cdot R d \phi & \left(\frac{\pi}{2}-\frac{\alpha}{2}\left\langle\theta<\frac{\pi}{2}\right)\right. \\
+\int_{\frac{\pi}{2}-\frac{\alpha}{2}}^{\theta} \frac{0.5 F \cos \phi-q R(1-\cos \beta)}{E A} \cdot \cos \phi \cdot R d \phi & \\
+\int_{\frac{\pi}{2}-\frac{\alpha}{2}}^{\theta} \frac{0.5 F R(1-\cos \phi)+M c-q R^{2}(1-\cos \beta)}{E I} \cdot R(1-\cos \phi) \cdot R d \phi &
\end{array}\right.
$$

Where $q$ was distributed load, $\mathrm{N} / \mathrm{m}$ and $F=2 \int_{0}^{\frac{\alpha}{2}} q \cos \theta \cdot R d \theta=2 q R \sin \frac{\alpha}{2}$. All the geometric parameters of annular ring and their values were shown in Table1.

Tab.1 The Basic Parameter of the Annular Ring

\begin{tabular}{cccccccc}
\hline Item & Force/ $F$ & $\begin{array}{c}\text { Contact } \\
\text { arc } \\
\text { Angle } / \alpha\end{array}$ & $\begin{array}{c}\text { Inner } \\
\text { diameter/D1 }\end{array}$ & $\begin{array}{c}\text { Diameter } \\
\text { of sectiond }\end{array}$ & $\begin{array}{c}\text { sectional } \\
\text { area/ } A\end{array}$ & $\begin{array}{c}\text { inertia } \\
\text { moment/I }\end{array}$ & $\begin{array}{c}\text { Elasticity } \\
\text { modulus } E\end{array}$ \\
\hline Value & $1000(\mathrm{~N})$ & $30\left(^{\circ}\right)$ & $52(\mathrm{~mm})$ & $3.5(\mathrm{~mm})$ & $4 \pi\left(\mathrm{mm}^{2}\right)$ & $4 \pi\left(\mathrm{mm}^{4}\right)$ & $206(\mathrm{Gpa})$ \\
\hline
\end{tabular}

The device was designed with the parameters above and the curve of each point's deformation displacement along with the angle was shown in Figure 3. 


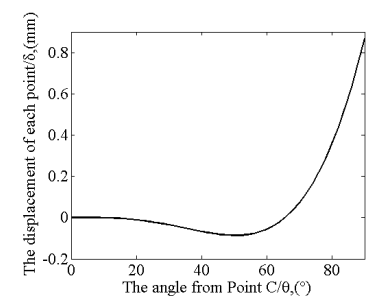

Fig.3 The Curve of Every Displacement in the Annular Ring

The figure showed that from Point $\mathrm{C}$ to Point $\mathrm{A}$, the curve kept stable first and then decrease slowly with the increase of the angle, finally increased quickly, because the annuar ring distorts under the stress of cutting edge shaft. The maximum deformation was in Point A, and $\delta_{A}=0.8670 \mathrm{~mm}$.

The Deformation of Annular Ring under the Changing Parameters.

Changing the parameters to study the deformation of the annular ring. Fistly, make the section diameter $3 \sim 4.5 \mathrm{~mm}$, it could get the curve of the effect of every displacement in the annular ring by the changing diameter of section $d$ in Figure $4 \mathrm{~A}$ ). Then maKe the inner diameter of the ring range from $45 \mathrm{~mm}$ to $60 \mathrm{~mm}$ to get the curve, which was shown Figure $4 \mathrm{~b}$ ).

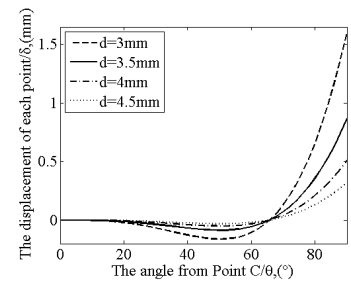

a) The effect of thesection diameter $d$

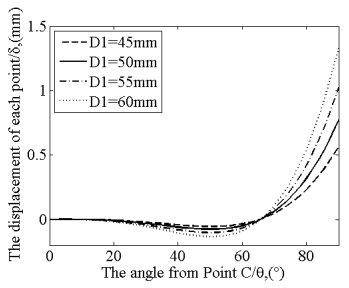

b) The effect of the inner diameter $D 1$

Fig.4 The Curve of the Effect of Every Displacement in the Annular Ring by the Changing Diameter of Section $d$ and Inner Diameter D1

What could be seen from the first graph was that when diameter of section $d$ was larger, the deformation displacement would be smaller and when inner diameter $D l$ was larger, the deformation displacement would be larger, that was because the rigidity of the annular ring was becoming large. Compared with Figures 4 and 5, it could be concluded that each deformation of the point on the annular ring reduced to a negative value first and then increased rapidly. The impact of the diameter of section $d$ was larger than the inner diameter $D 1$. That was because the diameter of section $d$ affects the rigidity more.

\section{The Finite Element Analysis of Annular Ring under Load}

The Analysis and Optimization of the Annular Ring.

According to the real situation of the device above, it added $1000 \mathrm{~N}$ on both sides of the annular ring uniformly and applied fixed constraint on the axial[8], then got the results shown in Figure 5.

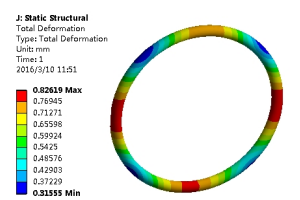

a) The deformation nephogram

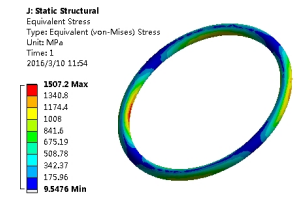

b) The stress nephogram

Fig.5 The Finite Element Analysis Result of Annular Ring

It shows that the annular ring had turn to ellipse under the force and it got the maximum stress 1507.2Mpa and maximum deformation $0.82619 \mathrm{~mm}$.

In order to optimize the deformation of the ring it set the Diameter of Section $d$ and Inner Diameter $D 1$ as the input parameters and maximum stress and maximum deformation as output ones to got the response surface of optimization, which was illustrated in Figure 6. 


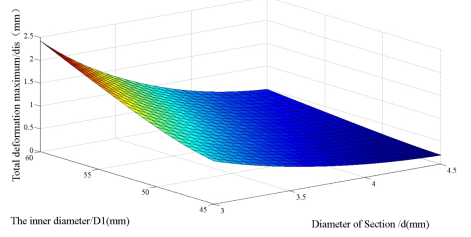

a) The Response Surface of deformation

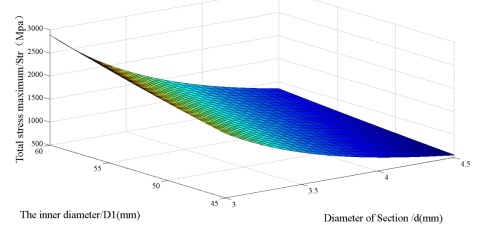

b) The Response Surface of stress

Fig.6 The Response Surface of Optimized Result of Annular Ring

From the figure above, it showed that the larger of the inner diameter $D 1$ and the smaller of the sectional diameter $d$, the larger of deformation displacement and stress will be, vice versa. This phenomenon came from the effect of the rigidity of the annular ring.

\section{The Mechanics Experiment of the Annular Ring}

\section{The Experiment Process.}

According to structure of part 8 and 11 in Figure 1 it designed and manufacture the testing device shown in Figure 6, it consisted of the pressured head, annular ring and the seat of pressured head. Among them, the annular with the 304 stainless steel was covered on the pressured head, which are made by the 38CrMoAlA after heat treatment to get HRC50 60.

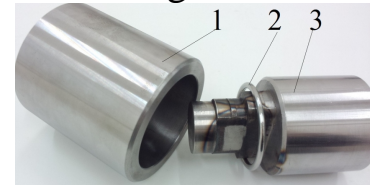

(1.The seat of pressured head 2.Annular ring 3.Pressured head)

Fig.7 The Device Using for Test

The equipment was Electro-mechanical Universal Testing Machines produced by Guangzhou Grandtry Testing Instruments LTD[9]. The product model was WD50-KE and ninth accuracy grade. The experiment process was following in Figure 8.

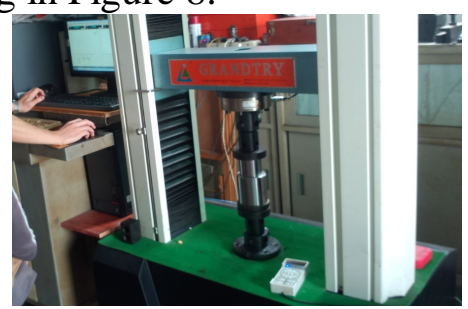

Fig.8 The Loading Process

Load on the pressure head slowly by the Electro-mechanical Universal Testing Machines When testing, transfer the displacement $x$ and the force $F_{a}$ of the pressure head to the computer with the sensor and then store and display the data. The technological parameters of the experiment as following the velocity $v=2 \mathrm{~mm} / \mathrm{min}$, the maximum pressure was $20 \mathrm{kN}$ and the environmental temperature was $20^{\circ} \mathrm{C}$.

\section{The Result Analysis of the Experiment.}

The inner diameter $\mathrm{Dl}$ of the annular ring using for the test were $52 \mathrm{~mm}$ and $40 \mathrm{~mm}$ and the diameter of section d were $3.5 \mathrm{~mm}, 4 \mathrm{~mm}$ and $4.5 \mathrm{~mm}$. Test several times to compare each other and some results of the test are shown in Fig 9. 


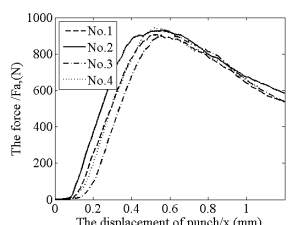

(a) $D 1=52 \mathrm{~mm}, \quad d=3.5 \mathrm{~mm}$

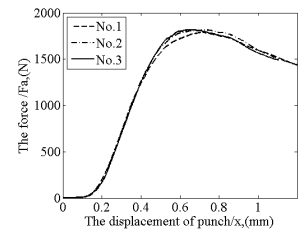

(b) $D 1=52 \mathrm{~mm}, d=4.5$

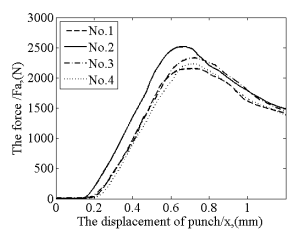

(c) $D 1=40 \mathrm{~mm}, d=3.5 \mathrm{~mm}$

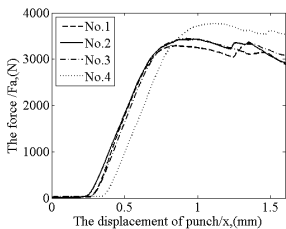

(d) $D 1=40 \mathrm{~mm}, d=4.5 \mathrm{~mm}$

Fig.9 The Result of the Experiment

As illustrated in Figure 10, in the experiment, the axial force of the annular ring equaled to the pressure $F_{a}$ of the pressure head added by the testing machine, and the radial force $F_{r}$ was the force to step over the Cutting edge shaft.
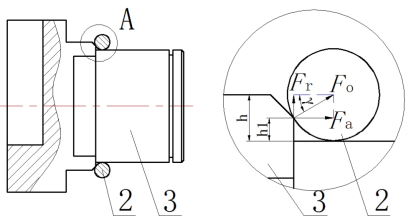

Fig.10The Diagram of Force between the Annular Ring and the Transitional Step of the Cutting Edge Shaft

It chosed the height of the step $h=1 \mathrm{~mm}, h_{1}=0.5 \mathrm{~mm}$, from the relation shown in Figure 9 , it got the function below.

$$
F_{r}=F_{a} \cdot \tan \gamma=F_{a} \cdot \frac{\frac{d}{2}-h_{1}}{\sqrt{\left(\frac{d}{2}\right)^{2}-\left(\frac{d}{2}-h_{1}\right)^{2}}}
$$

The result of the experiment compared with the calculating ones could be displayed as following in Table 2.

Tab.2 The Maximum Forces in the Experiment $(\mathrm{kN})$

\begin{tabular}{|c|c|c|c|c|c|}
\hline$\frac{d /(\mathrm{mm})}{D 1 /(\mathrm{mm})}$ & & . & 3.5 & 4 & 4.5 \\
\hline \multirow{7}{*}{52} & \multirow{6}{*}{ Experimental data } & $1 \#$ & 0.904 & 1.283 & 1.787 \\
\hline & & $2 \#$ & 0.916 & 1.139 & - \\
\hline & & $3 \#$ & 0.923 & 1.366 & 1.815 \\
\hline & & 4\# & 0.943 & 1.243 & 1.818 \\
\hline & & $F_{a}$ (average) & 0.922 & 1.258 & 1.807 \\
\hline & & $F_{r}$ & 0.941 & 1.426 & 2.237 \\
\hline & Calculating value & F & 0.949 & 1.575 & 2.452 \\
\hline \multirow{7}{*}{40} & \multirow{6}{*}{ Experimental data } & $1 \#$ & 2.158 & 3.068 & 3.284 \\
\hline & & $2 \#$ & 2.515 & 3.063 & 3.425 \\
\hline & & $3 \#$ & 2.331 & 3.024 & 3.443 \\
\hline & & $4 \#$ & 2.232 & 3.282 & 3.766 \\
\hline & & $F_{a}($ average $)$ & 2.309 & 3.109 & 3.480 \\
\hline & & $F_{r}$ & 2.332 & 3.503 & 4.307 \\
\hline & Calculating value & $F$ & 1.966 & 3.233 & 4.993 \\
\hline
\end{tabular}

\section{The Conclusion of the Experiment.}

Shown in Figure 8, the curves were smooth first and then increased rapidly nearly like linear, this was because the annular ring did not load by the pressure head at the beginning and then it deform elastically after loading. Kept loading to make the annular ring cross the steps, during the moment, the force of the annular ring would get the peak value. Later, the force would reduce quickly since the end of the 
deformation and the contact force would release, so the curves fell slowly. In this moment, the annular ring had already spanned the step of the cutting edge shaft.

From Figure 8(a)and(b)( or Figure 8 (c)、 and(d)), it indicated that when the inner diameter D1 was const, the larger of the section diameter $d$, the higher of the ordinate in the curve, which meant the peak force. That was illustrated the larger of the section diameter $d$ increased the stiffness of the annular ring. The same principle were shown in Figure 8(a)and(c)( or Figure 8 (b)、 and(d)).

Compared the data $F_{r}$ and $F$ in Table 2, it indicated that different annular rings would get different errors across the shaft shoulder, which were caused by the theoretical calculating value and the measured value. The errors came from the sliding friction between the cutting edge shaft and the annular ring, also it caused by the accuracy of manufacturing and the equipment itself. Of course, these errors should not be ignored. Hence, it is better to design to be a taper in the transition of the step and it would make stress smoothly. Besides, instead of making a chamfer, fillet the shaft shoulder to make sure the slide smoothly. Finally, improve the accuracy of the device in manufacturing.

For the single distribution network lines, when using the $\mathrm{K}$ level pole, the maximum permissible unbalance tension is about $1 \mathrm{kN}$ to amortized computation in the single line. So, when $D 1$ is $52 \mathrm{~mm}$ and $d$ is 3.5 to $4 \mathrm{~mm}$, the force value is about $1 \mathrm{kN}$ in Figure 2. Therefore, the data in this paper can be used to design and develop the device of "abandoning lines to protect electric poles".

\section{Conclusion}

The paper calculated the deformations and got the function curves of the annular rings under load by energy method, and then it make finite element analysis to optimal design the annular. The result showed that when the inner diameter $D 1$ was larger and the section diameter $d$ was smaller, the deformation of the annular ring was larger, vice versa. By the mechanical test, it got the characteristic of the annular ring when it was stepping over the cutting edge shaft and its stress. The result showed that the inner diameter $D 1$ was larger and the section diameter $d$ was smaller, the annular ring could step over easily. The experimental results meet well with the theoretical analysis, so the data could be used for calculation the annular ring and design the device. When manufacturing the annular ring, keep the consistency size of the annular rings will make sure of the precision in the device of "abandoning lines to protect electric poles". Otherwise, it can change the size of the annular ring to meet the requirement of any lever poles so the adaptation of the device is improved in this way. The paper provided the reference basis of the device of "abandoning lines to protect electric poles".

\section{References}

[1] D.S. Gazzana, G..D. Ferreira, A.S. Bretas,A.L. Bettiol, A.Carniato, L.F.N Passos, A.H.Ferreira, J.E.M. Silva. An integrated technique for fault location and section identification in distribution systems. Electric Power Systems Research, 2014.

[2] Ali, A, Khan, A.Q, Hussain, B, Raza, M.T, Arif, M.Fault modelling and detection in power generation, transmission and distribution systems[J]. IET Generation, Transmission \& Distribution,2015,9(16):278 291.

[3] Liang Zhen-hua, Xu Wei-dong. The device for abandoning lines to protect electric poles in transmission and distribution line and its assembly method[P]. Chinese patent, 201410102971.7, 2014-07-02

[4] Xie Wen-ping, Xiao Kai, Nie Ming. The device for protecting or abandoning lines to protect electric poles from typhoon in transmission and distribution line $[\mathrm{P}]$. Chinese patent, 201410070197.6, 2014-07-02.

[5] BROWN PT. Numerical analyses of uniformly loaded circular rafts on elastic layers of finite depth [J]. Geotechnique, 1969, 19(2):301-306. 
[6] He Ting-hui, Huang Xiao-jie, Lu Li-fang. Engineering Mechanics[M]. Guangzhou: South China university of technology press, 2010,8 .

[7] Aerospace and mechanical mechanics teaching research of Tongji university. Mechanics of materials [M]. Shanghai, Tongji university press, 2011, 2.

[8] $\mathrm{Hu}$, Dianyin, Wang, Rongqiao; Ren and Quanbin, etc. Finite element analysis of O-ring seal structure[J]. Journal of Beijing University of Aeronautics and Astronautics, 2005, 31(2):255-260.

[9] Information on http://www.grandtry.com/ 